\title{
Interactive comment on "Diurnal variation in the isotope composition of plant xylem water biases the depth of root-water uptake estimates" by Hannes P. T. De Deurwaerder et al.
}

\section{Anonymous Referee \#2}

Received and published: 20 February 2020

The manuscript by De Deurwaerder and colleagues challenges the idea that, in absence of precipitation or other rapid changes in climate, the water isotope composition of plant xylem should stay fairly constant over diurnal time scales or along stem height. Their analysis is based mostly on a model (!) of root water uptake and isotopic transport within the roots, up to the stem base. Their model considers that (1) the isotope composition of stem water at the base of a tree $\left(\delta^{2} H_{x}(0, t)\right)$ is the average isotope composition of soil water over the root zone, weighted by the fractional root water uptake rates at each depth (Eqs. 1 or 7 ) and (2) the isotope composition of stem water at any height $h\left(\delta^{2} H_{x}(h, t)\right)$ is the isotope composition of stem water at the base, delayed by Discussion paper the travel time $\tau$ of sap between stem base and height $h\left(\delta^{2} H_{x}(h, t)=\delta^{2} H_{x}(0, t-\tau)\right)$ 
(Eq. 9). Soil properties are used as boundary conditions that do not change over the day in terms of soil water potential and isotopic composition. With such model, they predict large diurnal variations of xylem water isotopes at stem base, but also large variations along the stem (see their Figs. 1 and 2). Based on this modelling exercise, and separate observations of the ${ }^{2} H /{ }^{1} H$ ratio in water extracted from tree stems and lianas at different heights within a tropical forest canopy, and showing some scatter sometimes larger than $\pm 3 \%$ o (the estimated error from water extraction and isotope analysis), they conclude that (1) the common assumption that the isotope composition of stem water is fairly constant over time is violated and (2) it can cause significant biases when using water isotopes to identify plant water origin.

I think it is good that the authors bring forward the point that xylem water may sometimes exhibit rather dynamic variations in its isotope composition. However, I am afraid the proposed model is inadequate and the dataset is too limited for illustrating this point. To me, the study does not prove anything; it shows that there are variations in the data and that there are variations in the model but there is no model-data comparison. Besides, variations in the data are not very large and can be explained by lots of other processes, and variations in the model are mostly caused by its lack of realism. These two points are explained in more details below.

The dataset accompanying this study only consists of a few water isotope data from tree stems and lianas collected over a couple of days. No soil water data is shown, or even sap flow or rooting depths. I doubt it is the best dataset to test the proposed theory, or draw any conclusion about plant water uptake. The data shown in Fig. 5 is interesting but it comes from another study (Zhao L, Wang L, Liu X, Xiao H, Ruan Y, Zhou M (2014) The patterns and implications of diurnal variations in the d-excess of plant water, shallow soil water and air moisture. Hydrology and Earth System Sciences, 18, 4129-4151). Many processes (stem evaporation, different proportions of storage tissues or even atmospheric vapour use) and measurement artefacts (during sampling and transport, water extraction, isotopic analysis...) could explain significant

Printer-friendly version

Discussion paper
Interactive comment

\section{2}


variations in the water composition of stems from trees and lianas of different statures. Accounting for uncertainties in the extraction and analysis is certainly not enough.

More importantly, I find the modelling analysis flawed and totally unrealistic. As explained above, the proposed model simulates water isotope gradients along the stem based on the average travel time of sap between two stem points (i.e. assuming the water isotope composition of xylem water at height $h$ is that at stem base at an earlier time corresponding to the travel time between stem base and height $h$ ). By doing so, the model neglects the mixing of water isotope by diffusion during water transport. If we neglect pit structure and consider vessels as regular pipes, the Péclet number $\wp$ that compares advection and diffusion is, using their notations: $\wp=S F_{V} h / D_{l}$. Taking an average sap flow velocity of $S F_{V}=0.3 \mathrm{mh}-1$ (see caption of Fig. 2) a typical height (diffusion length) $h$ of $1 \mathrm{~m}$ and a self-diffusion of liquid water of $D_{l}=2.510^{--9} \mathrm{~m}^{2} \mathrm{~s}^{-1}$ this leads a Péclet number $\wp$ around 30000 , i.e. high enough to justify neglecting (a posteriori) water mixing by diffusion. However, mixing with storage tissues also occurs and tree sap does not move like a slab. In their model, as soon as transpiration stops, root uptake stops and sap flow at any height stops too so that the $\delta^{2} H$ of xylem water at any height remains to its value at dusk over the entire night until the following morning plus the time delay $\tau$ (see for example Fig. 2a, the curve for $h=1.3 \mathrm{~m}$ ). In reality, at night, sap flow does not stop immediately because plant elastic tissues need to be replenished. Root uptake will continue until full replenishment of the elastic tissues is done. This will contribute to homogenisation of xylem water over night. Also when sap flow becomes small diffusion is not negligible anymore (low Péclet), which will reinforce the isotopic mixing by water diffusion. In other words, in the real world, xylem water should not exhibit large isotopic gradients along the stem such as shown in their Figs. 1 or 2. Mixing with storage tissues is briefly discussed (section 4.3.iii) but not in the same direction as above. If night-time mixing of xylem water in roots and stems was accounted for, this should strongly minimise the predicted diurnal variations of $\delta^{2} H_{x}(h, t)$, even at stem base. Not accounting for diurnal variations in soil conditions (water potential and isotopic composition) is also a strong limitation of the model.

Printer-friendly version

Discussion paper 
In conclusion, I find the argument raised by De Deurwaerder and colleagues not supported by their data nor by their model simulations. More realism would need to be brought to the model and the dataset should be complemented with additional information before drawing any conclusion on how variable the isotopic of xylem water in tree stems and lianas is over diurnal time scales or with height.

Interactive comment on Biogeosciences Discuss., https://doi.org/10.5194/bg-2019-512, 2020. 